\title{
Efficacy and safety of laparoscopic hepatectomy for hepatocellular carcinoma comorbid with cirrhosis
}

\author{
Yoshihiro Inoue ${ }^{1}$, Keisuke Yokohama², Hideko Ohama², Yusuke Tsuchimoto², Tetsuji Terazawa², Akira Asai², \\ Shinya Fukunishi ${ }^{2}$, Junji Okuda ${ }^{1}$, Kazuhide Higuchi ${ }^{2}$, Kazuhisa Uchiyama ${ }^{1}$ \\ ${ }^{1}$ Department of General and Gastroenterological Surgery, Osaka Medical College Hospital, Osaka, Japan \\ ${ }^{2}$ Second Department of Internal Medicine, Osaka Medical College Hospital, Osaka, Japan
}

Gastroenterology Rev 2020; 15 (3): 225-233

DOI: https://doi.org/10.5114/pg.2020.99039

Key words: liver cirrhosis, laparoscopic hepatectomy, hepatocellular carcinoma.

Address for correspondence: Yoshihiro Inoue MD, Department of General and Gastroenterological Surgery, Osaka Medical College Hospital, 2-7 Daigaku-machi, Takatsuki City, Osaka 569-8686, Japan, phone: +81 072(683)1221, fax: +81 072(685)2057, e-mail: sur129@osaka-med.ac.jp

\begin{abstract}
Introduction: Laparoscopic hepatectomy $(\mathrm{LH})$ is very difficult to perform in patients with cirrhosis because of the haemorrhagic and fibrotic nature of the liver, although there are various advantages to laparoscopic surgery.

Aim: To investigate the surgical outcomes, and efficacy and safety of LH versus open hepatectomy $(\mathrm{OH})$ for hepatocellular carcinoma $(\mathrm{HCC})$ resection.

Material and methods: A total of 112 patients with cirrhosis, who underwent hepatectomy, were analysed retrospectively. We investigated the safety and efficacy of LH for HCC with cirrhosis. Student's $t$ and $\chi^{2}$ tests, Mann-Whitney's $U$ test, Wilcoxon's signed-rank test, and Fisher's exact test were used in the statistical analysis.

Results: Seventy-one patients underwent $\mathrm{LH}$, and 41 underwent $\mathrm{OH}$. The conversion rate from $\mathrm{LH}$ to $\mathrm{OH}$ was $12.7 \%$. After propensity score matching, the estimated blood loss was significantly lower in the LH group than in the $\mathrm{OH}$ group ( $25 \mathrm{vs}$. $310 \mathrm{ml}$; $p<0.001)$, and there was a significant difference between the groups in the operative time $(p=0.091)$. The LH group had complication rates of $3.6 \%$ and $0 \%$ for refractory ascites and pleural effusion, respectively, while those were $17.9 \%$ and $10.7 \%$, respectively, in the $\mathrm{OH}$ group ( $p=0.019$ and $p=0.005$, respectively). The $\mathrm{LH}$ group had no mortality, whereas the $\mathrm{OH}$ group had a mortality rate of $10.7 \%(p=0.038)$. The postoperative length of stay was significantly longer in the LH group than in the $\mathrm{OH}$ group (9 days vs. 14 days) $(p=0.002)$.

Conclusions: $\mathrm{LH}$ can be performed safely for HCC with cirrhosis. More favourable results are achieved with $\mathrm{LH}$ than with $\mathrm{OH}$ in terms of surgical outcomes.
\end{abstract}

\section{Introduction}

In the last 20 years, dramatic advances have been made in laparoscopic surgery, based on its minimally invasive nature, as a replacement for conventional laparotomy procedures in many fields of surgery, including gastrointestinal surgery $[1,2]$. The impact of these advances has extended to hepatic resection procedures, and now many hepatic resections are performed laparoscopically [3].

We previously reported significantly favourable outcomes of laparoscopic partial hepatectomy compared to open partial hepatectomy, including reduced blood loss during surgery, reduced rate of superficial surgical site infection (SSI), and reduced postoperative inflammatory reaction $[1,4]$. These results are achieved due to ad- vances in the equipment used in laparoscopic surgery, and the improvement and standardisation of surgical procedures for laparoscopic hepatectomy. Although there are various advantages to laparoscopic surgery, including the magnifying effect, haemostatic effect of abdominal air pressure, and the minimal invasiveness of this procedure, complete laparoscopic minor hepatectomy $(\mathrm{LH})$ is very difficult to perform in patients with liver cirrhosis because of the haemorrhagic liver and fibrosis of the liver parenchyma [5].

\section{Aim}

In this study, we investigated the postoperative outcomes in a series of complete LHs performed in patients with advanced cirrhosis, as well as the safety and efficacy of this procedure. 


\section{Material and methods}

\section{Patient population and selection}

Laparoscopic hepatic resection was introduced in our hospital in 1998, and we gradually standardised the surgical procedure. Because a significant number of cases of laparoscopic hepatic resection had already accumulated by 2010, the procedures of laparoscopic hepatic resection were established. This study included patients who underwent this operation after 2010, when standardisation of the surgical procedure was established. A tumour size of $<10 \mathrm{~cm}$ was the main criterion that indicated $\mathrm{LH}$; tumour number or tumour location was not considered as a criterion for indicating LH. However, not more than five sites of hepatic resection were considered as an indication for $\mathrm{LH}$. Patients with main bile ductal involvement and/or metastasis to adjacent organs were not considered for $\mathrm{LH}$. Moreover, LH was not considered when any complication occurred after other surgical procedures.

Between January 6, 2010 and December 21, 2018 we conducted liver resection for liver tumours in 837 consecutive patients in Osaka Medical College Hospital, Takatsuki City, Japan. Minor hepatic resection was performed in 112 of these patients for hepatocellular carcinoma (HCC), who had a pathological diagnosis of F4 cirrhosis [6]. LH for HCC with cirrhosis was performed for 71 patients, and open minor hepatectomy $(\mathrm{OH})$ was performed for 41 patients. These 112 patients underwent hepatectomy with no other concomitant surgical procedure (i.e. colorectal). All patients were fully informed of the study design and provided their written informed consent to participate. This study was approved by the Ethics Committee on Clinical Investigation of Osaka Medical College Hospital (approval numbers 1828 and 1994).

We evaluated hepatic function using the ChildPugh classification [7] of liver dysfunction. Criteria to convert laparoscopic to open hepatic resection were as follows: (1) when the liver stumps of both preserved and resected sides could not be expanded adequately, (2) when intraoperative bleeding could not be controlled, (3) when blood loss exceeded $500 \mathrm{ml}$, (4) when the total time of the Pringle manoeuvre (hepatic blood flow occlusion) exceeded $120 \mathrm{~min}$, and (5) when intraoperative bile leakage indicated during the operation could not be improved. The patients who required conversion from $\mathrm{LH}$ to $\mathrm{OH}$ were analysed as part of the LH group.

\section{Surgical procedure}

In this series, all patients received potentially curative hepatic resection with the complete removal of the gross tumour with negative macroscopic margins.
All procedures were performed by three experienced hepatobiliary surgeons ( $\mathrm{Yl}, \mathrm{FH}$, and $\mathrm{KU}$ ) during the study period.

All procedures were performed with patients under general anaesthesia. The detailed open and laparoscopic surgical techniques routinely used in our department have been described in previous reports [4, 8-11]. Briefly, standard diagnostic and staging laparotomy was conducted. The liver was mobilised, and intraoperative ultrasonography (Prosound $\alpha 7$, Hitachi Aloka Medical Ltd., Tokyo, Japan) was routinely performed. Central venous pressure (CVP) was maintained at $0-3 \mathrm{~mm} \mathrm{Hg}$ during parenchymal transection. Parenchymal transection was achieved using a surgical tissue management system (Thunderbeat, Olympus Inc., Tokyo, Japan) and a Sonop 5000 ultrasonic dissector (Hitachi Aloka Medical, Ltd.). Small vessels were ligated or coagulated using a soft-coagulation system. Intraparenchymal control of major vessels was obtained with non-absorbable sutures, whereas biliary and vascular radicle division was accomplished with stapling devices or non-absorbable sutures. The hepatic pedicle was always isolated to enable performance of the Pringle manoeuvre when needed. Intermittent clamping was applied, with 15-minute clamping and 5-minute release periods. During the resection procedure, the surgical margin was carefully confirmed using intraoperative ultrasonography to obtain a surgical margin of 5-10 $\mathrm{mm}$ when possible.

\section{Data collection}

Data examined included preoperative factors, surgical factors, and pathological factors. Preoperative factors investigated were age, sex, American Society of Anaesthesiology (ASA) classification, body mass index (BMI), viral infection status, presence of diabetes mellitus, total bilirubin level, albumin level, prothrombin time (PT), platelet count, aspartate aminotransferase (AST) level, alanine aminotransferase (ALT) level, indocyanine green retention rate at $15 \mathrm{~min}$ (ICG-R15), Child-Pugh classification, and prognostic nutritional index. Surgical factors included the conversion rate, surgical duration, intraoperative blood loss, and blood transfusion requirements. Pathological factors included the size of the largest tumour, number of tumours, and surgical margin status. "R" classification denoted the absence or presence of a residual tumour after surgery [12]. R0 resection refers to excision of the tumour in one piece without violating the tumour plane or achieving negative margins after sequential re-excision of the involved margins. R1 resection involves a microscopically positive margin anywhere, and $\mathrm{R} 2$ resection involves one or more macroscopically positive margin(s) with visible tumour. 


\section{Postoperative evaluation}

The following parameters were evaluated: white blood cell (WBC) count, C-reactive protein (CRP) level, AST level, ALT level, platelet count, albumin level, total bilirubin level, PT, transfusion rate, pathological margins, postoperative complications, 30-day mortality, and hospital stay. Morbidity was graded according to Clavien-Dindo's classification [13, 14]. SSIs were defined according to the Centre for Disease Control's National Nosocomial Infection Surveillance system [15].

\section{Definitions}

Operative procedures were classified according to conventional terminology derived from the eight segments of the liver as per the Couinaud classification [16]. Anatomical resection was defined as resection of the neoplasm together with the portal vein related to the neoplasm and corresponding hepatic territory. Non-anatomical resection was defined as the resection of a lesion without regard to the segmental, sectional, or lobar anatomy.

Postoperative bile leakage and posthepatectomy liver failure were defined based on the criteria of the International Study Group of Liver Surgery [17, 18]. We defined massive ascites as ascites that could not be mobilised or as early recurrence that could not be satisfactorily prevented by medical therapy [19].

\section{Statistical analysis}

To minimise the effect of potential confounders on selection bias, propensity scores were generated using binary logistic regression analysis, which included the following variables: age, sex, ASA classification, BMI, hepatitis viral infection, diabetes mellitus, total bilirubin level, albumin level, PT, platelet count, AST level, ALT level, ICG-R15, Child-Pugh classification, number of tumours, largest tumour size, and tumour location. The choice of these variables was based on results of the univariate analysis and/or the known effect of specific factors on the selection of the type of intervention. Independent variables entered into the propensity model included the patients' preoperative information. Oneto-one matching between groups was accomplished using the nearest neighbour matching method, which was performed without replacement and using a calliper width of 0.2 standard deviations of the logit of the estimated propensity score. After propensity score matching (PSM), the two matched groups were handled as unpaired independent groups. Continuous variables are expressed as median \pm standard deviation. Results of univariate analysis were compared using Student's $t$ and $\chi^{2}$ tests, Mann-Whitney's $U$ test, Wilcoxon's signed- rank test, or Fisher's exact test, as appropriate. Factors that were found to be significant in the univariate analysis were included in multivariate logistic regression analysis to determine the adjusted odds ratios. Values of $p<0.05$ were considered significant. All statistical analyses were performed using JMP version 12 (SAS Institute, Inc., Cary, NC, USA).

\section{Results}

In the LH group, the laparoscopic procedure was successfully completed in 66 patients. However, 10 patients $(12.7 \%)$ were converted to $\mathrm{OH}$ because of bleeding from a hepatic vein branch, adhesion, and intraoperative bile leakage that could not be controlled laparoscopically and because the Pringle manoeuvre time exceeded $120 \mathrm{~min}$; they were included in the $\mathrm{OH}$ group. By PSM, 28 of 71 patients in the LH group could be matched with 28 of 41 patients in the $\mathrm{OH}$ group. The baseline characteristics of the matched study population (56 patients) are summarised in Table I. There were no significant differences in the demographic or operative characteristics between the groups.

Surgical outcomes are presented in Table II. After PSM, in cases in which hepatic resection was laparoscopic, the Pringle manoeuvre was performed in 17 of $28(60.7 \%)$ patients, and in open resections, the Pringle manoeuvre was performed in 11 of 28 (39.3\%) patients $(p=0.106)$. The estimated blood loss was significantly lower in the LH group ( $25 \mathrm{ml}$; range: $0-450 \mathrm{ml})$ than in the $\mathrm{OH}$ group (310 $\mathrm{ml}$; range: $0-1940 \mathrm{ml})(p<0.001)$. There was no significant difference between the two groups regarding the operative time $(p=0.091)$, although the $\mathrm{OH}$ group tended to have a longer operative time than the LH group.

Early-stage complications following surgical treatment including the incidences of SSIs and remote site infections within 30 days postoperatively were compared. The incidence of superficial incisional, deep incisional, and space/organ SSIs was not different between the two groups ( $p=0.313,1.000$, and 0.160 , respectively). The $\mathrm{LH}$ group had a complication rate of $3.6 \%$ for Clavien-Dindo grade IIla or higher, whereas the $\mathrm{OH}$ group had a complication rate of $35.7 \%(p=0.003)$. Moreover, the incidences of refractory ascites and respiratory complications including pleural effusion were significantly different between the two groups $(p=0.019$, $p=0.005$, and $p=0.038$, respectively). Overall, 8 (7.1\%) patients had in-hospital mortality: posthepatectomy liver failure (PHLF) in 7 patients and postoperative bile leakage in 1 patient. After PSM, the LH group had no mortality, whereas the $\mathrm{OH}$ group had a mortality rate of $10.7 \%(p=0.038)$. 
Table I. Patient demographic data

\begin{tabular}{|c|c|c|c|c|c|c|}
\hline \multirow[t]{2}{*}{ Parameter } & \multicolumn{3}{|c|}{ Before PSM } & \multicolumn{3}{|c|}{ After PSM } \\
\hline & LH & $\mathrm{OH}$ & $P$-value & LH & $\mathrm{OH}$ & $P$-value \\
\hline Number & 71 & 41 & & 28 & 28 & \\
\hline Conversion & $10(12.7 \%)$ & NA & NA & & & \\
\hline Age [years] & $71(49-93)$ & $72(42-83)$ & 0.195 & $73(54-93)$ & $72(42-81)$ & 0.177 \\
\hline $\operatorname{Sex}(M / F)$ & $47 / 24$ & $28 / 13$ & 0.820 & $19 / 9$ & $18 / 10$ & 0.778 \\
\hline ASA classification (1/2/3) & $6 / 31 / 34$ & $8 / 23 / 10$ & 0.088 & $2 / 12 / 14$ & $5 / 13 / 10$ & 0.236 \\
\hline Body mass index $\left[\mathrm{kg} / \mathrm{m}^{2}\right]$ & $23.9(17.1-34.9)$ & $23.8(18.1-37.4)$ & 0.784 & $23.6(17.1-34.9)$ & $24.1(18.1-31.3)$ & 0.769 \\
\hline Viral hepatitis infection (\%) & $53(74.7 \%)$ & $33(80.5 \%)$ & 0.481 & $23(82.1 \%)$ & $22(78.6 \%)$ & 0.737 \\
\hline Diabetes mellitus (\%) & $24(33.8 \%)$ & $17(41.5 \%)$ & 0.418 & $12(42.9 \%)$ & $13(46.4 \%)$ & 0.788 \\
\hline Total bilirubin [mg/dl] & $0.8(0.3-2.1)$ & $0.7(0.3-1.6)$ & 0.291 & $0.7(0.3-1.5)$ & $0.8(0.4-1.3)$ & 0.416 \\
\hline Albumin $[\mathrm{g} / \mathrm{dl}]$ & $3.8(2.8-4.5)$ & $4.0(2.9-4.5)$ & 0.205 & $3.8(3.1-4.5)$ & $4.1(2.9-4.5)$ & 0.537 \\
\hline Prothrombin time (\%) & $89(45-125)$ & $95(65-136)$ & 0.135 & $92(71-116)$ & $98(75-122)$ & 0.755 \\
\hline Platelet count $\left[\times 10^{4} / \mu \mathrm{l}\right]$ & $10.1(4.2-24.9)$ & $12.5(5.5-30.2)$ & 0.003 & $13.3(4.2-24.9)$ & $11.4(5.5-22.4)$ & 0.271 \\
\hline AST [U/l] & $46(15-173)$ & $45(16-96)$ & 0.171 & $35(15-164)$ & $45(16-86)$ & 0.719 \\
\hline $\operatorname{ALT}[\mathrm{U} / \mathrm{I}]$ & $38(7-186)$ & $30(10-131)$ & 0.138 & $29(8-154)$ & $41(10-85)$ & 0.493 \\
\hline ICGR-15 (\%) & $21.6(4.4-72.2)$ & $15.1(4.4-35.6)$ & 0.005 & $18.4(4.7-72.2)$ & $15.6(6.7-35.6)$ & 0.251 \\
\hline Child's grading (A/B) & $67 / 4$ & $35 / 6$ & 0.108 & $28 / 0$ & $27 / 1$ & 0.313 \\
\hline PNI & $45.3(38.4-55.8)$ & $44.2(37.0-57.6)$ & 0.966 & $46.9(40.3-55.8)$ & $50.6(41.7-57.6)$ & 0.590 \\
\hline Number of tumours & $1(1-5)$ & $1(1-4)$ & 0.712 & $1(1-4)$ & $1(1-4)$ & 0.850 \\
\hline Size of largest tumour [cm] & $2.2(0.6-6.0)$ & $3.0(1.0-18.9)$ & 0.002 & $2.4(0.6-6.0)$ & $2.4(1.0-4.8)$ & 0.673 \\
\hline Tumour location (\%): & & & 0.173 & & & 0.110 \\
\hline 1 & $2(2.8 \%)$ & $1(2.4 \%)$ & & $0(0 \%)$ & $2(7.1 \%)$ & \\
\hline ॥ & $7(9.9 \%)$ & $0(0 \%)$ & & $4(14.3 \%)$ & $0(0 \%)$ & \\
\hline III & $10(14.1 \%)$ & $3(7.3 \%)$ & & $7(25.0 \%)$ & $2(7.1 \%)$ & \\
\hline IV & $14(19.7 \%)$ & 7 (17.1\%) & & $4(14.3 \%)$ & $5(17.9 \%)$ & \\
\hline V & $6(8.5 \%)$ & $5(12.2 \%)$ & & $3(10.7 \%)$ & $4(14.3 \%)$ & \\
\hline $\mathrm{VI}$ & $15(21.1 \%)$ & $6(14.6 \%)$ & & $6(21.4 \%)$ & $3(10.7 \%)$ & \\
\hline VII & $8(11.3 \%)$ & $9(22.0 \%)$ & & $2(7.1 \%)$ & $6(21.4 \%)$ & \\
\hline VIII & $9(12.7 \%)$ & $10(24.4 \%)$ & & $2(7.1 \%)$ & $6(21.4 \%)$ & \\
\hline Tumor staging (I/II/III/IVA/IVB) & 21/38/10/1/1 & $7 / 21 / 11 / 1 / 1$ & 0.378 & $8 / 12 / 7 / 0 / 1$ & $9 / 14 / 5 / 0 / 0$ & 0.672 \\
\hline Number of hepatic resections & $1(1-4)$ & $1(1-5)$ & 0.450 & $1(1-4)$ & $1(1-5)$ & 0.644 \\
\hline
\end{tabular}

ASA - American Society of Anaesthesiology, NA - not applicable, PSM - propensity score matching, LH - laparoscopic hepatectomy, OH - open hepatectomy, AST - aspartate aminotransferase, ALT - alanine aminotransferase, ICGR-15 - Indocyanine green retention rate at 15 min, PNI - prognostic nutritional index.

The postoperative medical treatment was similar for the two groups, including intravenous electrolyte and balanced fluid solutions. Oral intake of fluid started on postoperative day 2 . The median postoperative duration of intravenous medicine was 5 days in both groups. The postoperative length of stay was significantly longer in the $\mathrm{OH}$ group (14 days; range: 9-71 days) than that in the LH group ( 9 days; range: $5-65$ days; $p=0.002$ ). 
Table II. Surgical procedures and results

\begin{tabular}{|c|c|c|c|c|c|c|}
\hline \multirow[t]{2}{*}{ Parameter } & \multicolumn{3}{|c|}{ Before PSM } & \multicolumn{3}{|c|}{ After PSM } \\
\hline & LH & $\mathrm{OH}$ & $P$-value & LH & $\mathrm{OH}$ & $P$-value \\
\hline Number & 71 & 41 & & 28 & 28 & \\
\hline Conversion & $10(12.7 \%)$ & NA & NA & & & \\
\hline Operative time [min] & $183(50-560)$ & $225(75-499)$ & 0.081 & $186(87-488)$ & $226(75-460)$ & 0.091 \\
\hline Blood loss [ml] & $50(0-1940)$ & $350(0-3590)$ & $<0.001$ & $25(0-450)$ & $310(0-1940)$ & $<0.001$ \\
\hline Blood transfusion (\%) & $23(32.4 \%)$ & $8(19.5 \%)$ & 0.142 & $4(14.3 \%)$ & $4(14.3 \%)$ & 1.000 \\
\hline Pringle manoeuvre (\%) & $35(49.3 \%)$ & $20(48.8 \%)$ & 0.668 & $17(60.7 \%)$ & $11(39.3 \%)$ & 0.106 \\
\hline Resected liver volume [g] & $35(3-330)$ & $115(5-1400)$ & 0.003 & $40(5-280)$ & $50(5-240)$ & 0.583 \\
\hline Surgical margin [mm] & $4(0-30)$ & $5(0-20)$ & 0.466 & $3(0-30)$ & $2(0-11)$ & 0.102 \\
\hline Curative resection, RO (\%) & $66(93.0 \%)$ & 35 (85.4\%) & 0.193 & $26(92.9 \%)$ & $25(89.3 \%)$ & 0.639 \\
\hline Postoperative complications (\%) & $20(28.2 \%)$ & $22(53.7 \%)$ & 0.007 & $8(28.6 \%)$ & $15(53.6 \%)$ & 0.057 \\
\hline Postoperative complications > IIIA (\%) & $7(9.9 \%)$ & $16(39.0 \%)$ & $<0.001$ & $1(3.6 \%)$ & $10(35.7 \%)$ & 0.003 \\
\hline Superficial incisional SSIs & $2(2.8 \%)$ & $2(4.9 \%)$ & 0.571 & $0(0 \%)$ & $1(3.6 \%)$ & 0.313 \\
\hline Deep incisional SSIs & $1(1.4 \%)$ & $0(0 \%)$ & 0.451 & $0(0 \%)$ & $0(0 \%)$ & 1.000 \\
\hline Organ/space SSIs & $5(7.0 \%)$ & $5(12.5 \%)$ & 0.335 & $1(3.6 \%)$ & $4(14.3 \%)$ & 0.160 \\
\hline Postoperative bile leakage & $3(4.2 \%)$ & $3(7.3 \%)$ & 0.484 & $1(3.6 \%)$ & $2(7.1 \%)$ & 0.553 \\
\hline PHLF: & $3(4.2 \%)$ & $5(12.2 \%)$ & 0.115 & $0(0 \%)$ & $3(10.7 \%)$ & 0.038 \\
\hline A & $0(0 \%)$ & $0(0 \%)$ & & $0(0 \%)$ & $0(0 \%)$ & \\
\hline B & $1(1.4 \%)$ & $0(0 \%)$ & & $0(0 \%)$ & $0(0 \%)$ & \\
\hline c & $2(2.8 \%)$ & $5(12.2 \%)$ & & $0(0 \%)$ & $3(10.7 \%)$ & \\
\hline Refractory ascites (\%) & $1(1.4 \%)$ & $8(19.5 \%)$ & $<0.001$ & $0(0 \%)$ & $5(17.9 \%)$ & 0.019 \\
\hline Respiratory complications (\%) & $1(1.4 \%)$ & $6(14.6 \%)$ & 0.005 & $0(0 \%)$ & $7(22.6 \%)$ & 0.005 \\
\hline Pleural effusion (\%) & $0(0 \%)$ & $11(15.9 \%)$ & 0.001 & $0(0 \%)$ & $3(10.7 \%)$ & 0.038 \\
\hline Mortality (\%) & $2(2.8 \%)$ & $6(14.6 \%)$ & 0.019 & $0(0 \%)$ & $3(10.7 \%)$ & 0.038 \\
\hline Postoperative length of stay [days] & $9(3-97)$ & $14(8-84)$ & 0.016 & $9(5-65)$ & $14(9-71)$ & 0.002 \\
\hline
\end{tabular}

PSM - propensity score matching, $\mathrm{LH}$ - laparoscopic repeat hepatectomy, $\mathrm{OH}$ - open repeat hepatectomy, $\mathrm{NA}$ - not applicable, SSI - surgical site infection, PHLF - posthepatectomy liver failure.

Postoperative AST levels peaked on day 1 and were almost normalised on day 7. Postoperative serum albumin levels, WBC counts, CRP levels, ALT levels, PTs, and platelet counts peaked on day 2 and then gradually normalised. Postoperatively, serum albumin levels, AST levels, ALT levels, WBC counts, and CRP levels, especially on the peak day, were significantly better in the LH group than in the $\mathrm{OH}$ group $(p=0.015, p=0.010, p=0.033$, $p=0.003$, and 0.003, respectively; Figures $1 \mathrm{~A}-\mathrm{F}$ ).

The 1-, 2-, 3-, and 5-year OS rates were 94.6\%, $91.7 \%, 79.5 \%$, and $63.6 \%$, respectively, with a median survival time of 27 months. The 1-, 2-, 3-, and 5-year recurrence-free survival (RFS) rates were $74.5 \%, 48.9 \%$,
$35.2 \%$, and $17.6 \%$, respectively. The $1-, 2-, 3-$, and 5 -year $\mathrm{OS}$ rates in the $\mathrm{LH}$ and $\mathrm{OH}$ groups were $100.0 \%$, $93.3 \%, 81.7 \%$, and $81.7 \%$ and $89.3 \%, 89.3 \%, 77.6 \%$, and $51.7 \%$, respectively $(p=0.260)$. The $1-, 2-, 3-$, and 5 -year RFS rates in the $\mathrm{LH}$ and $\mathrm{OH}$ group were $67.3 \%$, $45.6 \%, 28.5 \%$, and $28.5 \%$ and $82.2 \%, 53.3 \%, 41.5 \%$, and $0 \%$, respectively $(p=0.381)$.

\section{Discussion}

Factors suggested to affect the difficulty and invasiveness of hepatectomy include the operative time, blood loss, perioperative blood tests, and complications. In HCC complicated by cirrhosis, time is needed 
A

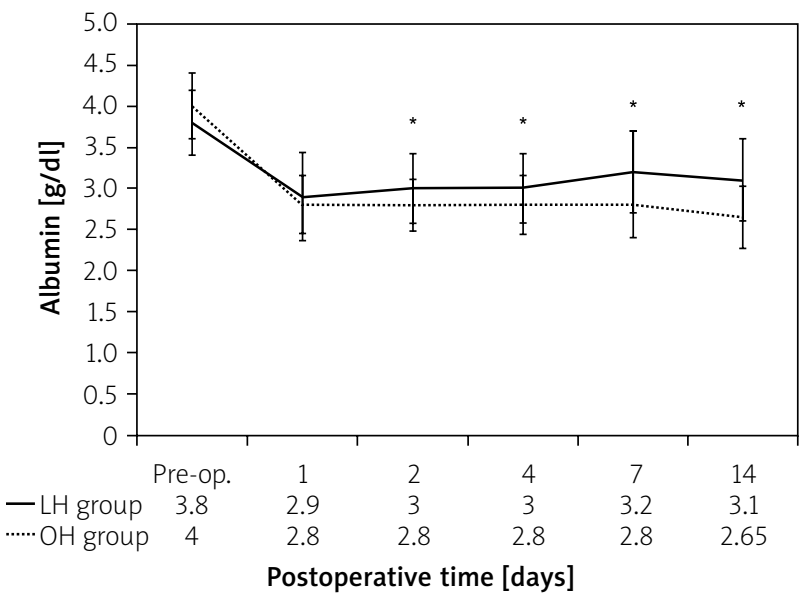

C

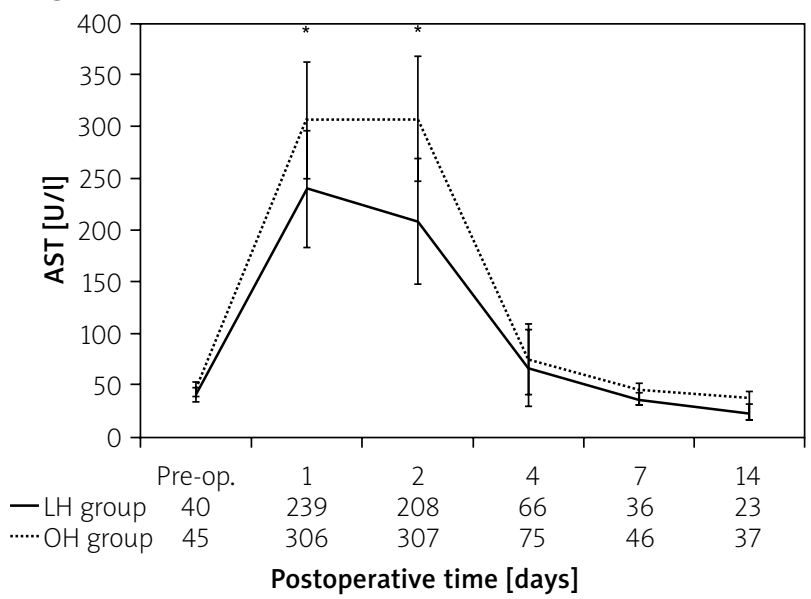

$E$

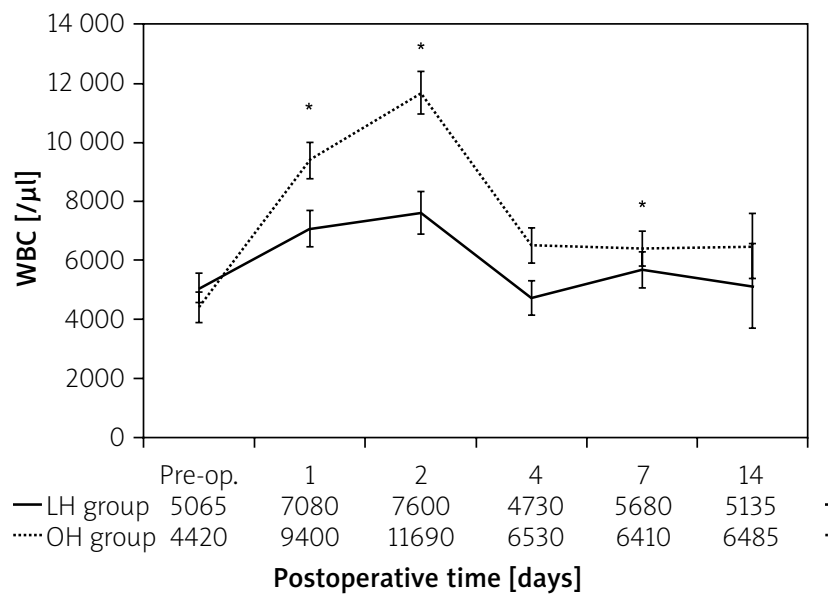

B

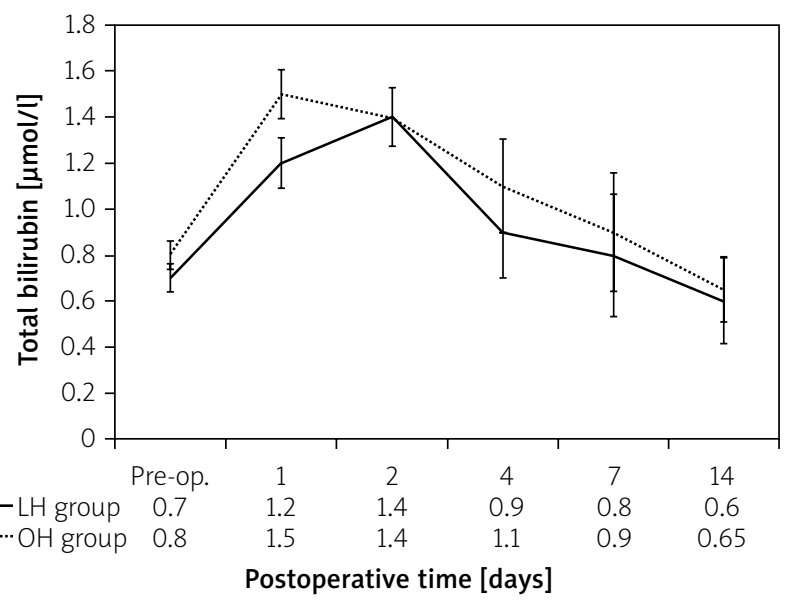

D

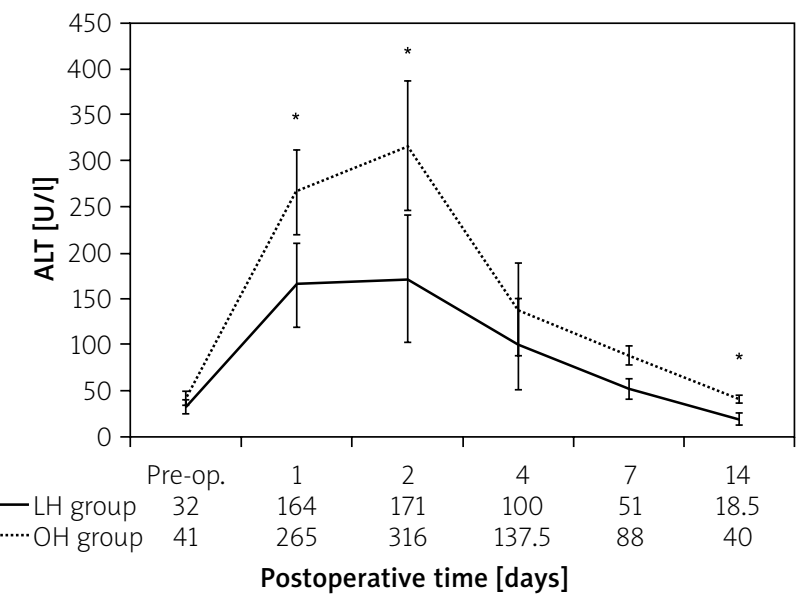

$\mathrm{F}$

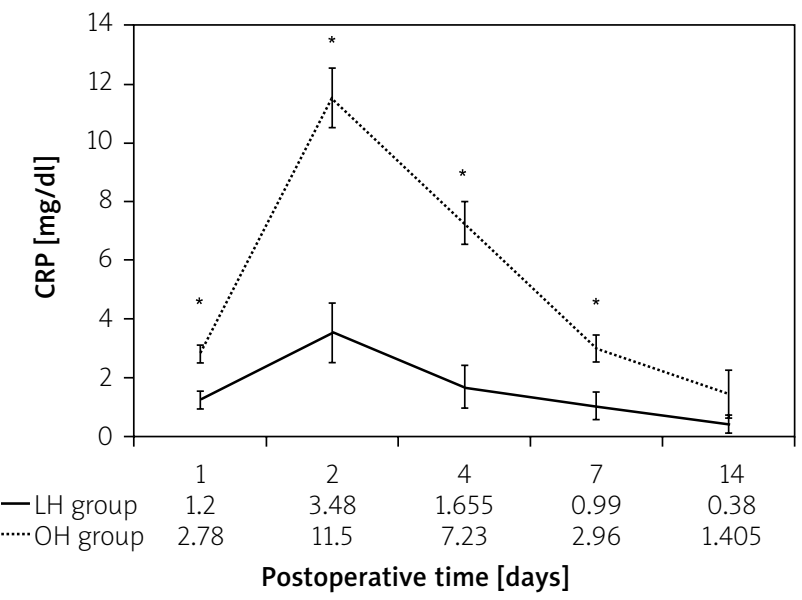

Figure 1. Postoperative changes in laboratory data. Postoperative serum albumin levels (A), total bilirubin levels (B), AST levels (C), ALT levels (D), WBC count (E), and CRP levels (F) in patients after hepatectomy. Postoperative serum albumin levels, AST levels, ALT levels, WBC counts, and CRP levels, especially on the peak day, are significantly better in the LH group than in the $\mathrm{OH}$ group $(p=0.015, p=0.010,0.033$, $p=0.003$, and $p=0.003$, respectively)

${ }^{*} P<0.05$. AST - aspartate aminotransferase, ALT - alanine aminotransferase, WBC-white blood cell, CRP - C-reactive protein, $L H$ - laparoscopic hepatectomy, $\mathrm{OH}$ - open hepatectomy. 
to expand the visual field because of the difficulty in mobilising the fibrotic liver, and dissection of the liver parenchyma does not always go as planned because of the hardness of the liver due to fibrosis. Many patients also have increased blood loss because of the difficulty in controlling blood loss during the surgery as well as decreased coagulability due to cirrhosis. The skill of the operator is also a factor. An increased incidence of PHLF is associated with increased perioperative blood loss, so it is essential to keep blood loss to a minimum. The incidence of PHLF in HCC with cirrhosis and postoperative complications consisting mainly of refractory ascites have tended to decrease in recent years, but preventing these conditions remains an important issue. Criteria for the indication of hepatic resection for $\mathrm{HCC}$ with cirrhosis have been investigated in numerous facilities to prevent early stage liver failure after liver resection.

Some recent reports [20-28] have confirmed the technical feasibility and safety of the laparoscopic technique for patients with liver tumours, but an ideal prospective, randomised study comparing laparoscopic and open hepatic resection has not yet been performed. This study retrospectively compared the degree of difficulty and invasiveness of laparoscopic and conventional open hepatic resections for liver tumours from the perspective of short-term outcomes in a single institution.

In our study, significantly better outcomes were found in the LH group, in terms of blood loss, postoperative complications, operative mortality rate, and postoperative length of stay. In terms of operative time, the laparoscopic group tended to have shorter operative times, but this was not significantly different between the groups. In the LH group, abdominal closure was not very time-consuming, whereas in the $\mathrm{OH}$ group, the skin incision was usually large, so abdominal closure often took an hour or more. A significant difference was also found in expansion of the visual field. Particularly for tumours located in segment 6,7 , or 8 , the right lobe of the liver must be fully mobilised to secure a visual field when dissecting the liver parenchyma. However, in cases with comorbid liver cirrhosis, a liver enclosed by the ribs has poor mobility, which often makes mobilisation difficult. Therefore, mobilisation was time consuming in the $\mathrm{OH}$ group, whereas in the laparoscopic group the liver could be approached from the lateral side by changing the laparoscopic insertion port, enabling the view required for dissection of the liver parenchyma to be secured with minimal mobilisation [8]. Thus, the difference in the operative times may be a reflection of these factors.

In terms of blood loss, perioperative blood loss during hepatectomy includes blood loss from the hepatic arteries, veins, and portal vein. Interrupting the inflow blood during dissection of the liver parenchyma, which is the main cause of perioperative blood loss, is one way of reducing the amount of perioperative blood loss [29]. In both groups, the inflow of blood was interrupted as much as possible, implementing what is known as the Pringle manoeuvre [30], thereby inhibiting arterial and portal vein haemorrhage. However, LH is more advantageous than $\mathrm{OH}$ in terms of venous haemorrhage. In the LH group, venous haemorrhage was inhibited by increasing abdominal air pressure, but there was no corresponding method available in the $\mathrm{OH}$ group [31]. This factor is thought to be the reason for the differences in our study's results. However, increasing intraabdominal pressure with pneumoperitoneum in patients with cardiac comorbidities during $\mathrm{LH}$ can reduce venous return, increase CVP because of reduced cardiac output, and lead to increased peripheral vascular resistance, which may conversely increase the risk of complications, so caution is needed [32, 33].

Lastly, in terms of perioperative blood test results and complications, although the laparoscopic group had no difference in the incidence of PHLF-related complications, the incidence of refractory ascites and respiratory complications including pleural effusion, and the operative mortality rate, were significantly lower. As reported previously, LH is a less invasive procedure than $\mathrm{OH}$ [34], and this fact is particularly notable in patients with comorbid cirrhosis, who have insufficient hepatic reserve. This is demonstrated by the fact that in perioperative blood test results, changes in short-term postoperative albumin levels were significantly lower in the $\mathrm{OH}$ group, whereas liver deviation enzymes, such as AST and ALT, and indicators of inflammatory responses, such as WBC and CRP, were significantly higher, indicating the significant difference in the invasiveness of the procedure.

$\mathrm{LH}$ was found to have various good outcomes in liver resection for patients with comorbid cirrhosis. However, this is not an affirmation of LH for all cases. In LH, there is restricted operation with forceps, and sufficient training is required to deal with the difficulty of setting detailed liver resection lines and expanding the field of view. Procedures associated with vascular reconstruction can be performed only in a limited number of facilities. There are still many issues associated with this procedure, including the need for caution in patients with comorbidities, such as heart disease, and it will take some time for these issues to be resolved effectively.

\section{Conclusions}

$\mathrm{LH}$ was not different compared to $\mathrm{OH}$ in terms of the operative time and incidence of postoperative liver failure in patients comorbid with cirrhosis, who have 
insufficient hepatic reserve. However, LH was associated with significantly lower perioperative blood loss, the incidence of postoperative complications (such as refractory ascites and pleural effusion), and operative mortality rate. Still, the number of cases in this study was small, and the study may have had several biases, including the location of the tumour, particularly with respect to the blood vessels; thus, it is difficult to claim that there is a high degree of evidence. Further randomised controlled trials and meta-analyses are needed.

\section{Conflict of interest}

The authors declare no conflict of interest.

\section{References}

1. Inoue $\mathrm{Y}$, Hayashi $M$, Tanaka R, et al. Short-term results of laparoscopic versus open liver resection for liver metastasis from colorectal cancer: a comparative study. Am Surg 2013; 79: 495-501.

2. Nord HJ. Laparoscopy - a historical perspective: are gastroenterologists going to reclaim it? Gastrointest Endosc 2008; 68: 67-8.

3. Ishizawa T, Gumbs AA, Kokudo N, et al. Laparoscopic segmentectomy of the liver: from segment I to VIII. Ann Surg 2012; 256: 959-64.

4. Inoue $\mathrm{Y}$, Suzuki $\mathrm{Y}$, Ota $\mathrm{M}$, et al. short- and long-term results of laparoscopic parenchyma-sparing hepatectomy for small-sized hepatocellular carcinoma: a comparative study using propensity score matching analysis. Am Surg 2018; 84: 230-7.

5. Ban $\mathrm{D}$, Tanabe $\mathrm{M}$, Ito $\mathrm{H}$, et al. A novel difficulty scoring system for laparoscopic liver resection. J Hepatobiliary Pancreat Sc 2014; 21: 745-53.

6. Desmet VJ, Gerber M, Hoofnagle, et al. Classification of chronic hepatitis: diagnosis, grading and staging. Hepatology 1994; 19: 1513-20.

7. Pugh RN, Murray-Lyon IM, Dawson JL, et al. Transection of the oesophagus for bleeding oesophageal varices. Br J Surg 1973; 60: 646-9.

8. Inoue $\mathrm{Y}$, Suzuki Y, Fujii K, et al. Laparoscopic liver resection using the lateral approach from intercostal ports in segments VI, VII, and VIII. J Gastrointest Surg 2017; 21: 2135-43.

9. Inoue Y, Suzuki Y, Fujii K, et al. Laparoscopic hepatic resection using extracorporeal pringle maneuver. J Laparoendosc Adv Surg Tech A 2018; 28: 452-58.

10. Inoue $\mathrm{Y}$, Ishii $\mathrm{M}$, Tsuchimoto $\mathrm{Y}$, et al. Comparison of resection site of standardized laparoscopic hepatic tumor resection. Videosurgery Miniinv 2018; 13: 333-41.

11. Inoue $\mathrm{Y}$, Fujii K, Ishii M, et al. Volumetric and functional regeneration of remnant liver after hepatectomy. J Gastrointest Surg 2019; 23: 914-21.

12. International Union Against Cancer (UICC). Sobin LH, Gospodarowicz MK, Wittekind C (eds.). TNM classification of malignant tumours. $7^{\text {th }}$ edition. Wiley-Blackwell, New York 2009.

13. Dindo D, Demartines N, Clavien PA. Classification of surgical complications: a new proposal with evaluation in a cohort of
6336 patients and results of a survey. Ann Surg 2004; 240 : 205-13.

14. Clavien PA, Barkun J, de Oliveira ML, et al. The Clavien-Dindo classification of surgical complications: five-year experience. Ann Surg 2009; 250: 187-96.

15. Mangram AJ, Horan TC, Pearson ML, et al. Guideline for prevention of surgical site infection, 1999. Centers for Disease Control and Prevention (CDC) Hospital Infection Control Practices Advisory Committee. Am J Infect Control 1999; 27: 97-132.

16. Couinaud C. Surgical anatomy of the liver. Several new aspects. Chirurgie 1986; 112: 337-42.

17. Koch M, Garden J, Padbury R, et al. Bile leakage after hepatobiliary and pancreatic surgery: a definition and grading of severity by the International Study Group of Liver Surgery. Surgery 2011; 149: 680-8.

18. Rahbari NN, Garden OJ, Padbury R, et al. Posthepatectomy liver failure: a definition and grading by the International Study Group of Liver Surgery (ISGLS). Surgery 2011; 149: 713-24.

19. Arroyo V, Gines P, Gerbes AL, et al. Definition and diagnostic criteria of refractory ascites and hepatorenal syndrome in cirrhosis. International Ascites Club. Hepatology 1996; 23: 164-76.

20. Uchiyama K, Ueno M, Ozawa S, et al. Combined use of contrast-enhanced intraoperative ultrasonography and a fluorescence navigation system for identifying hepatic metastases. World J Surg 2010; 34: 2953-9.

21. Ishizawa T, Gumbs AA, Kokudo N, et al. Laparoscopic segmentectomy of the liver: from segment I to VIII. Ann Surg 2012; 256: 959-64.

22. Gaujoux S, Kingham TP, Jarnagin WR, et al. Single-incision laparoscopic liver resection. Surg Endosc 2011; 25: 1489-94.

23. Okumura S, Goumard C, Gayet B, et al. Laparoscopic versus open two-stage hepatectomy for bilobar colorectal liver metastases: a bi-institutional, propensity score-matched study. Surgery 2019; 166: 959-66.

24. Hasegawa Y, Nitta H, Tatahara T, et al. Pure laparoscopic living donor hepatectomy using the Glissonean pedicle approach (with video). Surg Endosc 2019; 33: 2704-9.

25. Belli G, Fantini C, D’Agostino A, et al. Laparoscopic liver resection without Pringle Maneuver for HCC in cirrhotic patients. Chir Ital 2005; 57: 15-25.

26. Noda T, Eguchi H, Wada H, et al. Short-term surgical outcomes of minimally invasive repeat hepatectomy for recurrent liver cancer. Surg Endosc 2018; 32: 46-52.

27. Di Sandro S, Bagnardi V, Najjar M, et al. Minor laparoscopic liver resection for hepatocellular carcinoma is safer than minor open resection, especially for less compensated cirrhotic patients: propensity score analysis. Surg Oncol 2018; 27: 722-9.

28. Memeo R, de'Angelis N, Compagnon P, et al. Laparoscopic vs. open liver resection for hepatocellular carcinoma of cirrhotic liver: a case-control study. World J Surg 2014; 38: 2919-26.

29. Zhang Y, Yang H, Deng X, et al. Intermittent Pringle maneuver versus continuous hemihepatic vascular inflow occlusion using extra-glissonian approach in laparoscopic liver resection. Surg Endosc 2016; 30: 961-70.

30. Pringle JH. Notes on the arrest of hepatic hemorrhage due to trauma. Ann Surg 1908; 48: 541-9.

31. Jayaraman $\mathrm{S}$, Khakhar $\mathrm{A}$, Yang $\mathrm{H}$, et al. The association between central venous pressure pneumoperitoneum, and ve- 
nous carbon dioxide embolism in laparoscopic hepatectomy. Surg Endosc 2009; 23: 2369-73.

32. Tran TB, Worhunsky DJ, Spain DA, et al. The significance of underlying cardiac comorbidity on major adverse cardiac events after major liver resection. HPB 2016; 18: 742-7.

33. Nguyen NT, Wolfe BM. The physiologic effects of pneumoperitoneum in the morbidly obese. Ann Surg 2005; 241: 219-26.

34. Inoue $Y$, Suzuki Y, Ota $M$, et al. short- and long-term results of laparoscopic parenchyma-sparing hepatectomy for small-sized hepatocellular carcinoma: a comparative study using propensity score matching analysis. Am Surg 2018; 84: 230-7.

Received: 16.09.2019

Accepted: 1.10 .2019 